\title{
Multi-CAMPUS TEACHING IN A CANADIAN ENGINEERING ConteXt: Assessing Presence USING The CoI SurveY
}

\author{
Christoph Sielmann*, Casey Keulen, Shun-Fu Hu \\ Faculty of Applied Sciences, The University of British Columbia \\ Faculty of Education, The University of British Columbia \\ *Corresponding Author: christoph.sielmann@ubc.ca
}

\begin{abstract}
Multi-campus synchronous teaching using teleconferencing involves teaching to a class in-person and remotely, simultaneously. As an approach to postsecondary learning, it can offer students greater variety, access to remote experts, and opportunities to collaborate across regions. There are significant challenges to successfully managing a multi-campus course, where ongoing observation and evaluation of student experience is important in guiding pedagogical practice. Herein we explore learning experiences of students who attended a course taught in a multi-campus format as part of a new dual-campus engineering program offered at the University of British Columbia. We chose a Community of Inquiry (CoI) surveying tool to assess student experience by examining their perceptions on teaching, social, and cognitive presence at both campuses.
\end{abstract}

Data collected and analyzed with a Multivariate Analysis of Variance show a clear disparity between perceptions of Teaching Presence between the two campuses, with significance in both the Design \& Organization and Direct Instruction CoI subcategories. The ease of performing a CoI survey and assessing its results renders this approach to continuous improvement feasible for regular evaluation and continuous improvement within the Bahmani and Hjelsvold conceptual framework for multi-campus course development. The study was undertaken as part of continuous improvement within the engineering program, with results used to develop and inform multi-campus synchronous teaching best practices in a Canadian engineering context.

Keywords: Community of Inquiry, multi-campus, dual campus, ICT, presence, student experience, survey, information and communication technologies.

\section{INTRODUCTION}

Multi-campus instruction involves the synchronous teaching of two or more student cohorts located at separate campuses where one cohort is in the physical presence of the instructor and the remaining cohorts attend through information and communication technologies (ICT). It is seen by some as a step on the road to fully remote instruction, and by others as an ideal compromise that benefits both students and educational institutions [1]. These benefits include greater access to a diversity of expertise, improved operational efficiencies, and a more versatile learning experience [1], [2].

Despite over two decades of case studies, a broadly accepted formalism on the design, delivery, and maintenance of courses and programs taught using a multicampus synchronous teaching format has yet to be validated. Two challenges explored by Bahmani and Hjelsvold in their recent work proposing such a framework are sensitivity to context and the importance of ongoing observation. The conceptual framework incorporates components from a transtheoretical model of change within an instruction design model. A simplified form of the model is shown in Figure 1. A key component to the proposed model is the Observation and Evaluation of the course during delivery, where ongoing observation "...helps the course and program stakeholders to gain awareness about any problem ... and help them to find a solution...”[3]

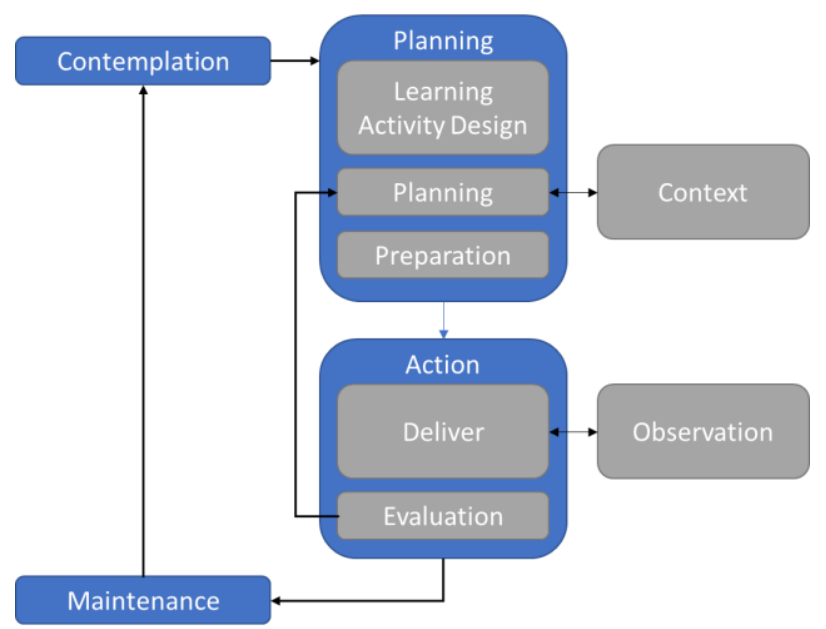

Figure 1: Simplified Conceptual Framework

Best practices must be responsive and adaptable to enhance student experience within the course and program, while considering the context specific to each campus involved, and using appropriate pedagogy and technology to promote equity and ensure accessibility for all students. Ongoing observation of the student experience and 
assessment is necessary to ensure that the student experience remains positive through perceived teaching presence, social presence, and cognitive presence, and that students continue to experience their learning equitably. A failure to manage student sentiment within a multi-campus setting can lead to a rapid and unequal deterioration in the student learning experience and classroom community [1], [4], [5]. We build on the proposed conceptual framework by examining one methodology suitable for easy deployment within multi-campus engineering courses that can facilitate periodic observation of key presence indicators.

Various methodologies have been used to explore student and instructor experiences in multi-campus courses, both during and after the course, including focus groups [6]-[9], interviews [1], [8], [10], [11], journaling [11], [12], absolute grades [13], and surveys [8], [14]. An appropriate tool for assessing student experience in all multi-campus courses within an engineering context that can readily satisfy the Observation requirement in the proposed conceptual framework must be simple to deploy, consistent, low impact, equitable for all parties at all campuses, demonstrate ease of analysis, and minimize burden on the students.

We chose a Community of Inquiry (CoI) surveying tool to assess student experience by examining their perceptions on teaching, social, and cognitive presence at both campuses [15], [16]. The CoI framework, originally developed by Garrison et al. [16], has been successfully used to assess student experience in other case studies [6], [14], [17] through interviews and focus groups.

The CoI framework separates student experience into three overlapping "Presence" indicators: Teaching Presence, Social Presence, and Cognitive as shown in Figure 2 [16]. By separating information collected into categories and subcategories, the CoI provides an effective tool for gaining insight into different dimensions of the student experience, whereas using another quantitative methodology such as absolute grades provides insights into the learning and assessment process, but is less diagnostically relevant for understanding student perceptions of social and teaching presence in the classroom. Also, to serve the overarching purpose of this paper, which is for an institution to better support students and instructors, the CoI framework provides a particularly well-integrated network for multiple common and trending themes in teaching and learning simultaneously. These themes include student engagement, course facilitation, course technologies and institutional support for students, as systematically reviewed by Martin, Sun, and Westine [18].

Teaching Presence (TP) concerns itself with the perceived connection formed between the student and the educator, and is subdivided into Design and Organization, Facilitation, and Direct Instruction. A lack in Teaching Presence can cause feelings of mistrust, disengagement, or mistrust within the students. Teaching presence drops with a lessened perception of educator presence within the classroom, reduced quality in student-instructor interactions, or a diminished educator influence in and outside the classroom.

Social Presence (SP) corresponds to a feeling of a cohesive student community, or "...the ability of participants in a community of inquiry to project themselves socially and emotionally, as 'real' people (i.e. their full personality), through the medium of communication being used." [16] Social Presence can be explored through Affective Expression, Open Communication, and Group Cohesion subcategories. Technical issues and cohort inequity factor strongly in maintaining a strong social presence within and outside the classroom.

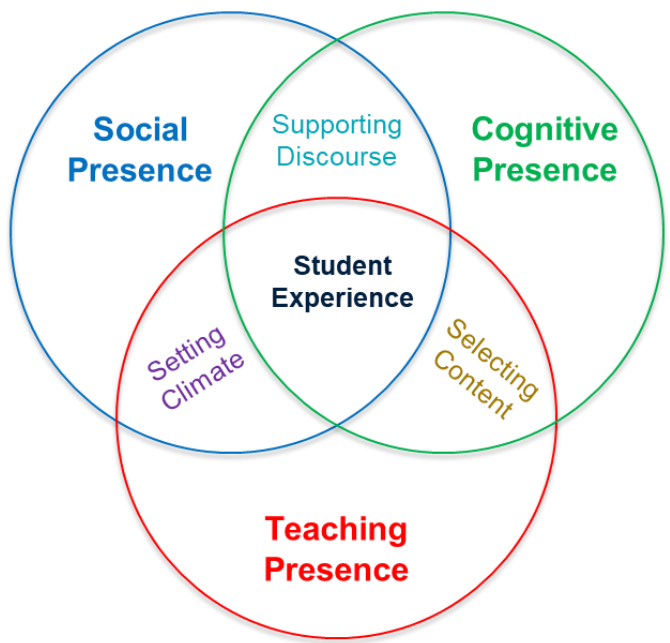

Figure 2: Community of Inquiry Framework

Social Presence (SP) corresponds to a feeling of a cohesive student community, or "...the ability of participants in a community of inquiry to project themselves socially and emotionally, as 'real' people (i.e. their full personality), through the medium of communication being used." [16] Social Presence can be explored through Affective Expression, Open Communication, and Group Cohesion subcategories. Technical issues and cohort inequity factor strongly in maintaining a strong social presence within and outside the classroom.

Cognitive Presence (CP) is the tendency for students to feel intellectually and emotionally engaged with the course. Subcategories include Triggering Event, Exploration, Integration, and Resolution, which represent a sequential process of inquiry and learning students should undertake frequently throughout a class to remain attentive and learn effectively. Content delivery can strongly influence $\mathrm{CP}$, where non-interactive, didactic teaching styles tend to reduce $\mathrm{CP}$ within the classroom. Multicampus instruction must emphasize engaging and 
interactive pedagogy to overcome the "flat" appearance of the course content as perceived by remote cohorts.

Although the CoI Framework has been successfully used in other multi-campus case studies, the original formalism requires focus groups or interviews as well as the application of a coding methodology to derive conclusions. A CoI survey is easy to deploy, complete, and analyze, though the results may not be as informative or dynamic as an interview or focus group. Surveys are traditionally used to facilitate course observation and evaluation in many existing engineering programs.

To satisfy the requirement proposed by Bahmani and Hjelsvold to engage in ongoing observation and evaluation of the student experience, a validated CoI-based survey was used in this analysis [15]. Examining student perception of presence using a validated survey tool allows for simplified, ongoing observation and periodic reassessment of the student learning experience.

We explore two questions in this study:

1. Can elements of Bahmani's and Hjelsvold's proposed framework be applied once a course has been developed as part of pedagogical continuous improvement?

2. Is the CoI survey an effective tool for highlighting variations in perceptions of presence between local and remote cohorts in a multi-campus engineering setting?

\section{METHODOLOGY}

\subsection{Overview}

This study aims to provide a starting point of a series of attempts to develop and inform teaching best practices in a Canadian engineering context, specifically in a MultiCampus Synchronous Teaching through Information and Communication Technologies (MCST-ICT) setting. Specifically, we ask whether and in which (sub)areas of Presence (see Introduction) there is a need to develop courses in formats tailored to MCST-ICT.

\subsection{Data Source and Procedure}

The final sample consisted of sixteen students who had completed a new dual-campus engineering course at the University of British Columbia. Of the 16 students, 5 were from the local and 11 were from the remote campus. All fully completed the CoI survey, the main measurement instrument employed in this study, without any missing data.

The final sample was out of thirty-two (total students enrolled) invitations sent to students via e-mail after the completion of the course. Participants had been assured of full confidentiality and absence of consequences if they declined participation during informed consent.
The course was developed and deployed using conventional teaching best practices non-specific to the MCST-ICT setting and was delivered through bi-weekly lectures using a modern ICT classroom. Participants in the local cohort were situated with the instructor in person and those in the remote cohort saw identical lecture content through synchronous streaming with ICT in a remote campus in the same time zone. Participants in the local cohort also knew the instructor from another course and had exposure to a different curriculum due to differences in the program between the campuses. A teaching assistant (TA) facilitated the class in the remote cohort. For both cohorts, assignments and other course materials were provided through an online course tool, Canvas.

\subsection{The Adapted Community of Inquiry Survey (CoI) and Data}

The current study used a validated version of the CoI survey by Swan et al. [15], which assessed Teaching Presence (the perceived connection formed between the student and the educator, TP), Social Presence (a feeling of a cohesive student community, SP) and Cognitive Presence (the tendency for students to feel intellectually and emotionally engaged with the course, CP), each of which also consists of subcategories. Table 1 summarizes the categories and subcategories with example questions of the CoI [15] with the full survey provided in Appendix A. The survey used is version 14 available from the University of Athabasca [19]. Validation of the survey produced eigenvalues from principal component analysis that were used to adjust our results and better isolate TP, SP, and CP components from the data [20].

Table 1: Summary of CoI survey categories [19]

\begin{tabular}{|c|c|c|}
\hline Category & Subcategory & Sample Questions \\
\hline \multirow[t]{3}{*}{$\begin{array}{l}\text { Teaching } \\
\text { Presence }\end{array}$} & $\begin{array}{c}\text { Design and } \\
\text { Organization }\end{array}$ & \multirow{2}{*}{$\begin{array}{l}\text { The instructor clearly } \\
\text { communicated important } \\
\text { course topics. }\end{array}$} \\
\hline & Facilitation & \\
\hline & $\begin{array}{c}\text { Direct } \\
\text { Instruction }\end{array}$ & $\begin{array}{l}\text { The instructor provided } \\
\text { feedback in a timely } \\
\text { fashion. }\end{array}$ \\
\hline \multirow[t]{3}{*}{$\begin{array}{l}\text { Social } \\
\text { Presence }\end{array}$} & $\begin{array}{c}\text { Affective } \\
\text { Expression }\end{array}$ & \multirow{2}{*}{$\begin{array}{l}\text { I was able to form dist- } \\
\text { inct impressions of some } \\
\text { course participants. }\end{array}$} \\
\hline & $\begin{array}{c}\text { Open } \\
\text { Communication }\end{array}$ & \\
\hline & $\begin{array}{l}\text { Group } \\
\text { Cohesion }\end{array}$ & $\begin{array}{l}\text { Online discussions help } \\
\text { me to develop a sense of } \\
\text { collaboration. }\end{array}$ \\
\hline \multirow[t]{4}{*}{$\begin{array}{l}\text { Cognitive } \\
\text { Presence }\end{array}$} & $\begin{array}{c}\text { Triggering } \\
\text { Event }\end{array}$ & \multirow{4}{*}{$\begin{array}{l}\text { Course activities piqued } \\
\text { my curiosity. } \\
\text { I can describe ways to } \\
\text { test and apply the } \\
\text { knowledge created in } \\
\text { this course. }\end{array}$} \\
\hline & Exploration & \\
\hline & Integration & \\
\hline & Resolution & \\
\hline
\end{tabular}


The survey was presented to students using the Qualtrics platform where a Likert scale (1 to 5) was mapped from Strongly Disagree to Strongly Agree. There were no reverse-coded items.

\section{RESULTS AND DISCUSSION}

\subsection{Omnibus MANOVA on Overall Presence}

To identify the need to specifically develop teaching best practices for MCST-ICT, a Multivariate Analysis of Variance (MANOVA), which is commonly used to address the intercorrelations among the dependent variables, was conducted using $\mathrm{R}$ [21]. The independent variable was Campus Cohort \{Remote, Local\}; The test was nondirectional, with hypothesis not specifying which one of the two campus sites should have higher means, using a Type I error rate (alpha) of .05. The dependent variables were TP, SP, and CP scores, obtained by taking the arithmetic means of participants' responses (from $1-5$ ) across all forms of presence. The item-factor mapping (i.e., TP, SP and CP) was supported by previous evidence [15]. Students required a median of 262.5 seconds to complete the survey. The analysis was conducted using $\mathrm{R}$ [21].

The omnibus MANOVA showed an overall difference in combined presence or general student experience $(\mathrm{F}(1,14)=11.06$, Pillai $=0.73, \mathrm{p}<.001)$ at alpha $=0.05$. Thus, we proceed with post-hoc analysis to locate where in the TP, SP and CP the differences lie.

\subsection{Post-hoc Analysis For TP, SP, and CP}

As seen in Table 2, post-hoc independent-sample t-tests showed that participants at the remote campus reported significantly lower scores on Teaching Presence vs. the local campus (Remote: $M=2.39, S D=0.73$; Local $M=$ $4.31, S D=0.61$ ) only at an alpha level of $.05 / 3=.0167$ (the division by 3 being the number of pair-wise comparisons $C_{2}^{3}$, to ensure a family-wise alpha of .05).

The $.98(1-.0167)$ confidence intervals (CIs) showing the differences between the two campuses demonstrated the same information, with the CIs for only TP not spanning across the value of 0 . There is no significant difference in perceived Social Presence and Cognitive Presence between the two cohorts, indicating an absence of evidence that they are primary subjects of concern among the students in the course.

\subsection{Subcategory Analysis of TP}

Subcategory means and standard deviations are indicated by Figure 3 . The results anecdotally match the analysis from 3.1, where a clear divide between Teaching Presence exists at both campuses. TP was perceived as very positive by the local cohort and very negative by the remote cohort, even in contrast to the other categories and subcategories. It is therefore an excellent target for further analysis to better isolate the cause of the difference and develop targeted improvements.

Three subcategories were similarly examined using post-hoc independent-sample t-tests on previous post-hoc independent-sample tests with results shown in Table 3. To ensure an overall alpha level of .05 , we used a stringent alpha $.05 / 9=.0056$ (three pair-wise comparisons each following each of the three comparisons in the previous post-hoc analysis, resulting in 9 controlled comparisons). Of the three subcategories, both "Design and Organization" and "Direct Instruction" demonstrate significance through both $\mathrm{p}$ and confidence interval (not spanning across 0). For this reason, both Direct Instruction as well as Design and Organization should be the focus of continuous improvement strategies applied to this course to improve equity between the cohorts.

Although there is no significant difference in either SP or $\mathrm{CP}$ between the two cohorts at alpha $=.05 / 3=.0167$, the mean values of each for are quite low, meaning that strategies could also be explored to improve presence in these two categories at both campuses.

\subsection{Discussion}

The small class size limits the generalizability of the data set, but it is adequate for exploring the effectiveness of the CoI survey as a tool for highlighting opportunities to improve the course under consideration. The survey results and corresponding analysis indicate that improvements should be made in the following areas:

1. Equity in Teaching Presence should be improved, particularly in Design and Organization and Direct Instruction.

Table 2: Summary of Post-hoc Analysis by Presence and Campus cohort Following a Significant omnibus MANOVA Result (not shown in the table, see text in 3.1).

\begin{tabular}{|l|c|c|c|c|c|}
\hline \multicolumn{1}{|c|}{ Category } & Remote Cohort & Local Cohort & .98 CI Lower Bound & .98 CI Upper Bound & p \\
\hline Teaching Presence & $2.385(0.734)$ & $4.308(0.608)$ & 0.904 & 2.942 & $<.001 *$ \\
\hline Social Presence & $3.071(0.895)$ & $3.333(1.264)$ & -1.809 & 2.334 & .69 \\
\hline Cognitive Presence & $3.053(0.684)$ & $3.950(0.573)$ & -0.061 & 1.855 & .023 \\
\hline
\end{tabular}

* Statistical significance at alpha $=.05 / 3=.0167$. The division by 3 is to ensure a family-wise error at .05 . The confidence intervals (.98 CI Upper and Lower bounds) show the upper and lower bounds of the difference between the two cohorts such that if the interval contains 0 then there is no evidence in perception of presence. 


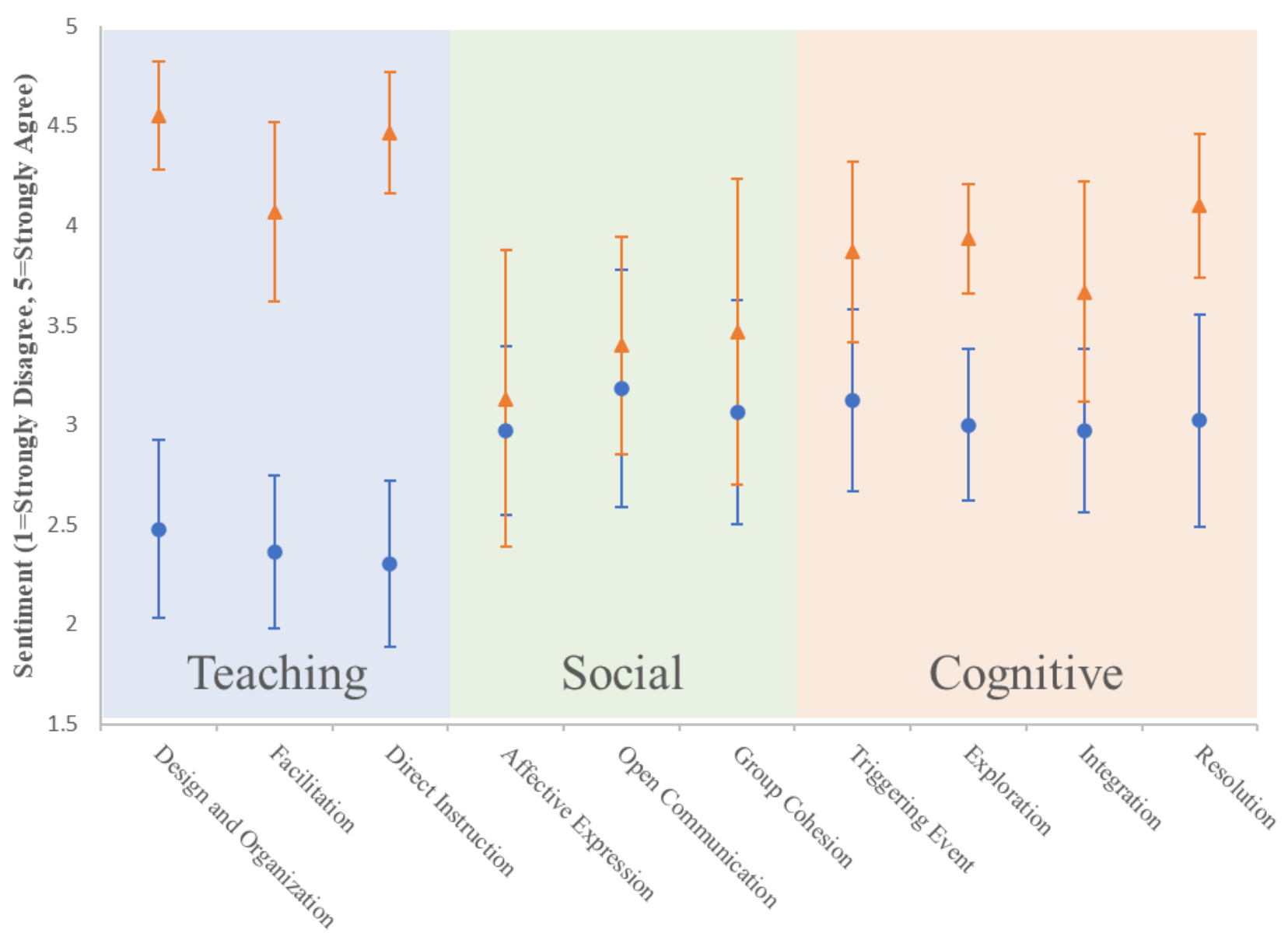

Presence Category

Figure 3: Subcategory Summary of Results. Error bars represent the standard deviation for each subcategory.

2. General improvements should be made to Social Presence and Cognitive Presence, though both cohorts perceived these two indicators similarly.

Equity between cohorts is exceptionally important in a multi-campus setting and improvements to equity should be prioritized [10]. Design and Organization of the course pertains to how effectively the instructor communicated course topics, goals, instructions on how to participate in learning activities, and course deadlines. A perceived inequity can occur if the remote cohort has less access to the instructor before and after class, especially when topics of course assessments are discussed. Instructors may participate in candid discussions with students "off camera", which is not possible for remote cohorts. Solutions that can improve equity in Design and Organization include:

- Posting all assessments, assignments, and schedules asynchronously online through a course delivery application.

Table 3: Post-hoc independent-sample t-tests for subcategory summary of Teaching Presence.

\begin{tabular}{|l|c|c|c|c|c|}
\hline \multicolumn{1}{|c|}{ Subcategory } & Remote Cohort & Local Cohort & $\begin{array}{c}\text {.99 CI Lower } \\
\text { Bound }\end{array}$ & $\begin{array}{c}\text {.99 CI Upper } \\
\text { Bound }\end{array}$ & p \\
\hline Design and Organization & $2.477(0.891)$ & $4.55(0.542)$ & 0.861 & 3.284 & $<.001^{*}$ \\
\hline Facilitation & $2.364(0.77)$ & $4.067(0.902)$ & -0.157 & 3.563 & .009 \\
\hline Direct Instruction & $2.303(0.836)$ & $4.467(0.606)$ & 0.884 & 3.443 & $<.001 *$ \\
\hline
\end{tabular}

* Statistical significance at alpha $=.05 / 9=.0056$. The division by 9 is to ensure a family-wise error at .05 . The confidence intervals (.99 CI Upper and Lower bounds, .99 coming from $1-.0056=.9944)$ show the upper and lower bounds of the difference between the two cohorts such that if the interval contains 0 then there is no evidence in perception of presence. 
- Ensuring that no "off camera" discussions take place with students or allocating time for discussion with each cohort before and after each class.

- Providing reminders of key deadlines to all cohorts at the start of each class.

Direct Instruction involves focussing discussions in class on topics that promote learning, offering feedback through assessments and class discussion, and offering feedback in a timely fashion. Equity in Direct Instruction is challenging to achieve in a multi-campus setting if the TA facilitating remote cohorts lacks adequate training to provide engaging, ongoing, and useful direction and feedback during synchronous activities. The following strategies can help to improve Teaching Presence in Direct Instruction:

- Specialized training focussing on facilitation and instruction for teaching assistants involved in multi-campus courses.

- Providing a common interface for all assessment submissions.

- Treating all assessments such as assignments and exams as common, without segregating student work product by cohort. All students should have equal access to feedback from the course instructor.

Despite the small class size and number of survey respondents, statistically significant results were acquired highlighting opportunities for improvement within the course. Combined with the reusability of the survey tool and the short median time for students to complete the survey of under 5 minutes, the CoI survey is an effective tool for gaining insights into the student experience within a multi-campus engineering course.

Although the course was not developed using the framework proposed by Bahmani and Hjelsvold, adding a CoI survey instrument presents minimal disruption to the flow of the course, and it was useful after the course concluded as part of the Evaluation and Maintenance components of the course.

Successful multi-campus teaching requires educators present in all sessions at all campuses [7]. Furthermore, research shows that there is a need for responsive and efficient technical support services [8]. Communication outages or difficulty using ICT equipment can significantly impact TP for remote cohorts as the course instructor becomes less accessible.

Improvements to Social Presence are also indicated by the data. Social Presence is established through student community, group cohesion, comfort in the classroom setting, and a feeling of inclusion. A tendency in multicampus courses is for barriers to form between cohorts, which may become adversarial. Building a consistent community of inquiry across campuses can be achieved through group and team activities that encourage mixing of students in each cohort. Asynchronous activities to build intercampus student community can effectively build positive social presence in the classroom.

\section{CONCLUSIONS}

Reframing a new multi-campus course at the University of British Columbia within the conceptual model proposed by Bahmani and Hjelsvold highlighted the importance of ongoing observation and evaluation of the student experience during and after course delivery. Although not initially designed with the framework, adapting course evaluation to fit within the framework using a survey tool to measure student experience toward evaluating equity and presence in a multi-campus engineering context provided insights into opportunities for course and program improvement.

Analysis of the survey results demonstrated significant differences in perceived Teaching Presence, especially in Design and Organization as well as Direct Instruction, highlighting these areas for prioritized improvement within the "Planning" stage of the framework to achieve greater equity between local and remote cohorts. Further improvements to Social Presence are also generally indicated from the survey results, though this is not specific to a cohort.

The results collected from the CoI survey are consistent with other case studies in literature demonstrating the effects of pursuing multi-campus teaching without an established framework or formalism. As a tool for contributing to the Observation and Evaluation components in Bahmani and Hjelsvold's framework, the CoI survey was easy to implement, quick to use by students, and provided useful insights for course maintenance and improvement. The survey may be used on an ongoing basis during multi-campus course delivery to rapidly isolate issues and provide a means for corrective action.

Pedagogical recommendations were made on the basis on the survey results to improve equity in Teaching Presence and generally improve Social Presence. Future work will evaluate the effectiveness of these recommendations within the existing context and the ongoing efficacy of using $\mathrm{CoI}$ to assess presence in multicampus engineering courses.

\section{Acknowledgements}

We would like to thank the students who completed the survey and the Faculty of Applied Sciences for their guidance and financial support in pursuing this work. This work was also supported in part by the Institute for Scholarship of Teaching and Learning (ISoTL) at the University of British Columbia.

The primary focus of this work is quality assurance and improvement within a specific course and program. A standard, vetted survey instrument was used and TCPS2 ethical guidelines were followed in engaging student participation, maintaining anonymity in data collection and storage, minimizing risk to vulnerable students, and promoting beneficence. Results of the student survey and 
this work will be applied directly and immediately to improving the course and program.

\section{References}

[1] T. Lawson, C. Comber, J. Gage, and A. CullumHanshaw, "Images of the future for education? Videoconferencing: a literature review," Technology, Pedagogy and Education, vol. 19, no. 3, pp. 295-314, Oct. 2010, doi: 10.1080/1475939X.2010.513761.

[2] M. Hastie, I.-C. Hung, N.-S. Chen, and Kinshuk, "A blended synchronous learning model for educational international collaboration," Innovations in Education and Teaching International, vol. 47, no. 1, pp. 9-24, Feb. 2010, doi: 10.1080/14703290903525812.

[3] A. Bahmani and R. Hjelsvold, "Work-inProgress: Towards a Conceptual Framework for MultiCampus Course Development," in 2020 IEEE Global Engineering Education Conference (EDUCON), Apr. 2020, pp. 1584-1588, doi: 10.1109/EDUCON45650.2020.9125193.

[4] J. Gage, T. Beardon, and M. Nickson, "Can videoconferencing contribute to teaching and learning? The experience of the Motivate Project," 2002.

[5] M. Doggett, "The Videoconferencing Classroom: What Do Students Think?," Architectural and Manufacturing Sciences Faculty Publications, Jan. 2007.

[6] E. Szeto, "Bridging the Students' and Instructor's Experiences: Exploring Instructional Potential of Videoconference in Multi-Campus Universities," Turkish Online Journal of Educational Technology - TOJET, vol. 13, no. 1, pp. 64-72, Jan. 2014.

[7] A. Divanoglou, K. Chance-Larsen, J. Fleming, and M. Wolfe, "Physiotherapy student perspectives on synchronous dual-campus learning and teaching," AJET, vol. 34, no. 3, Jul. 2018, doi: 10.14742/ajet.3460.

[8] M. Freeman, "Video Conferencing: a Solution to the Multi-campus Large Classes Problem?," British Journal of Educational Technology, vol. 29, no. 3, pp. 197-210, Jul. 1998, doi: 10.1111/1467-8535.00064.

[9] T. Andrews and G. Klease, "Challenges of multisite video conferencing: The development of an alternative teaching/learning model," AJET, vol. 14, no. 2, Oct. 1998, doi: 10.14742/ajet.1902.

[10] R. Hjelsvold and A. Bahmani, "Challenges in Repurposing Single-Campus Courses to Multi-Campus Settings," Laring om laring, vol. 3, no. 1, Art. no. 1, Apr. 2019, Accessed: Sep. 14, 2020. [Online]. Available: https://www.ntnu.no/ojs/index.php/lol/article/view/3033.

[11] E. Szeto, "Transforming Learning and Teaching in Higher Education: The Impact of ICT on Pedagogy, Peer Interaction and Support in a Networked Virtual Learning Environment," The International Journal of Learning: Annual Review, vol. 17, no. 11, pp. 205-214, 2011, doi: 10.18848/1447-9494/CGP/v17i11/47344.
[12] D. Knipe and M. Lee, "The quality of teaching and learning via videoconferencing," British Journal of Educational Technology, vol. 33, no. 3, pp. 301-311, Jun. 2002, doi: 10.1111/1467-8535.00265.

[13] J. Jiang, L. Zhao, and J. Qian, "On education quality control issues for Sino-France hybrid engineer diploma," in IEEE EDUCON 2010 Conference, Apr. 2010, pp. 481-485, doi: 10.1109/EDUCON.2010.5492539.

[14] M. Yamada, "The role of social presence in learner-centered communicative language learning using synchronous computer-mediated communication: Experimental study," Computers \& Education, vol. 52, no. 4, pp. 820-833, May 2009, doi: 10.1016/j.compedu.2008.12.007.

[15] K. Swan, J. Richardson, P. Ice, D. Garrison, M. Cleveland-Innes, and J. B. Arbaugh, "Validating a Measurement Tool of Presence in Online Communities of Inquiry," E-Mentor, vol. 2, Jan. 2008.

[16] D. R. Garrison, T. Anderson, and W. Archer, "Critical Inquiry in a Text-Based Environment: Computer Conferencing in Higher Education," The Internet and Higher Education, vol. 2, no. 2, pp. 87-105, Mar. 1999, doi: 10.1016/S1096-7516(00)00016-6.

[17] C. Niyizamwiyitira, "Teaching using videoconferencing: approaches and challenges," Lärarlärdom, pp. 103-123, 2015, doi: 10.15626/lld.201506.

[18] F. Martin, T. Sun, and C. D. Westine, "A systematic review of research on online teaching and learning from 2009 to 2018," Comput Educ, vol. 159, p. 104009, Dec. 2020, doi: 10.1016/j.compedu.2020.104009.

[19] "CoI Survey | CoI." https://coi.athabascau.ca/coimodel/coi-survey/ (accessed Feb. 28, 2021).

[20] J. B. Arbaugh et al., "Developing a community of inquiry instrument: Testing a measure of the Community of Inquiry framework using a multi-institutional sample," The Internet and Higher Education, vol. 11, no. 3-4, pp. 133-136, Jan. 2008, doi: 10.1016/j.iheduc.2008.06.003.

[21] R. C. Team, "R: A language and environment for statistical computing," 2013.

\section{Appendix A: COMPlete CoI SURVEY}

The following is the complete, validated CoI Survey used in our analysis. [19].

\section{Teaching Presence}

\section{Design \& Organization}

1. The instructor clearly communicated important course topics.

2. The instructor clearly communicated important course goals.

3. The instructor provided clear instructions on how to participate in course learning activities.

4. The instructor clearly communicated important due dates/time frames for learning activities. 


\section{Facilitation}

5. The instructor was helpful in identifying areas of agreement and disagreement on course topics that helped me to learn.

6. The instructor was helpful in guiding the class towards understanding course topics in a way that helped me clarify my thinking.

7. The instructor helped to keep course participants engaged and participating in productive dialogue.

8. The instructor helped keep the course participants on task in a way that helped me to learn.

9. The instructor encouraged course participants to explore new concepts in this course.

10. Instructor actions reinforced the development of a sense of community among course participants.

\section{Direct Instruction}

11. The instructor helped to focus discussion on relevant issues in a way that helped me to learn.

12. The instructor provided feedback that helped me understand my strengths and weaknesses relative to the course's goals and objectives.

13. The instructor provided feedback in a timely fashion.

\section{Social Presence}

\section{Affective expression}

14. Getting to know other course participants gave me a sense of belonging in the course.

15. I was able to form distinct impressions of some course participants.

16. Online or web-based communication is an excellent medium for social interaction.

Open communication

17. I felt comfortable conversing through the online medium.

18. I felt comfortable participating in the course discussions.

19. I felt comfortable interacting with other course participants.

\section{Group cohesion}

20. I felt comfortable disagreeing with other course participants while still maintaining a sense of trust.

21 . I felt that my point of view was acknowledged by other course participants.

22. Online discussions help me to develop a sense of collaboration.

\section{Cognitive Presence}

Triggering event

23. Problems posed increased my interest in course issues.

24. Course activities piqued my curiosity.
25. I felt motivated to explore content related questions.

\section{Exploration}

26. I utilized a variety of information sources to explore problems posed in this course.

27. Brainstorming and finding relevant information helped me resolve content related questions.

28. Online discussions were valuable in helping me appreciate different perspectives.

Integration

29. Combining new information helped me answer questions raised in course activities.

30. Learning activities helped me construct explanations/solutions.

31. Reflection on course content and discussions helped me understand fundamental concepts in this class.

\section{Resolution}

32. I can describe ways to test and apply the knowledge created in this course.

33. I have developed solutions to course problems that can be applied in practice.

34. I can apply the knowledge created in this course to my work or other non-class related activities. 